\title{
Commentary \\ The effect of antimicrobial resistance on patient outcomes: importance of proper evaluation of appropriate therapy
}

\author{
Mitchell J Schwaber ${ }^{1}$ and Yehuda Carmeli ${ }^{2}$
}

\author{
${ }^{1}$ National Center for Infection Control, Israel Ministry of Health, 6 Weizmann St., Tel Aviv 64239, Israel \\ ${ }^{2}$ Department of Epidemiology and Preventive Medicine, Tel Aviv Medical Center, 6 Weizmann St., Tel Aviv 64239, Israel
}

Corresponding author: Mitchell J Schwaber, mitchells@tasmc.health.gov.il

Published: 12 January 2009

This article is online at http://ccforum.com/content/13/1/106

(C) 2009 BioMed Central Ltd

Critical Care 2009, 13:106 (doi:10.1186/cc7136)

See related research by Depuydt et al., http://ccforum.com/content/12/6/R142

\begin{abstract}
The impact of antimicrobial resistance on patient outcomes can be effectively measured only if the appropriateness of the antimicrobial therapy received is properly measured. Definition of appropriate therapy should include not only in vitro susceptibility but also the clinical adequacy of the antibiotic used, taking into account the pathogen isolated, the site of infection, known pharmacokinetic and pharmacodynamic properties of the drug, and dosing. In the absence of these data, the effect of delay or absence of appropriate therapy in patients infected with resistant bacterial pathogens is subject to confounding, and the true effect of resistance on outcomes may be obscured.
\end{abstract}

Do infections with antibiotic-resistant pathogens yield worse outcomes than similar infections in similar patients with susceptible pathogens? The practical importance of knowing the answer to this question relates both to infection control that is, are the considerable resources and inconveniences required to contain the spread of multi-drug-resistant (MDR) bacteria justified by reduced morbidity and mortality? - and to treatment decisions - that is, should empirical antimicrobial therapy be modified in the face of resistance? A number of reasons that MDR pathogens may be associated with worse outcomes can be offered, including increased virulence of MDR strains of bacteria, inferior therapeutic options for MDR bacteria owing to restrictions posed by resistance profiles, and delay in the initiation of appropriate therapy owing to the resistance. The first reason offered is pathogen-related, the second and third treatment-related.

In a recent issue of Critical Care, Depuydt and colleagues addressed this question in a cohort of patients with ventilatorassociated pneumonia (VAP) [1]. In a carefully designed study using state of the art microbiological and statistical methods, they found that resistance is not an independent predictor of 30-day in-hospital mortality. This conclusion, though reported in the past as well [2], contrasts with those of several recent studies that found that antimicrobial resistance does independently predict poor outcomes in VAP [3-5]. In light of Depuydt and colleagues' findings, should we revise our perceptions regarding the deleterious effects of multi-drug resistance on patient outcomes?

Leaving aside for a moment the conclusions of the authors, let us consider whether the data collected offer us the opportunity to compare infections caused by MDR versus nonMDR pathogens. Regarding the putative pathogen-related cause for worse outcomes - increased virulence of MDR pathogens compared with their non-MDR counterparts - we can draw no conclusions from this study. In order to compare virulence, the study would have to include data on the bacteria themselves. In the absence of these data, we might be able to infer something about the bacteria if presented with a head-to-head comparison of a specific pathogenrelated infection, that is, VAP caused by MDR Pseudomonas versus VAP caused by non-MDR Pseudomonas. The study design, which compares cases of VAP caused by MDR pathogens with cases of VAP caused by non-MDR pathogens, does not allow for organism-related conclusions.

Regarding the potential therapy-related causes for poor outcomes - inferior antibiotics and delay in effective therapy here too Depuydt and colleagues' study does not allow for comparison, because its definition of effective therapy does not account for treatment that is microbiologically effective but clinically problematic. Take, for example, a patient with VAP caused by methicillin-susceptible Staphylococcus aureus who is treated with vancomycin rather than an antstaphylococcal beta-lactam agent. According to the study

MDR, multi-drug-resistant; VAP, ventilator-associated pneumonia. 
definitions, this patient received appropriate therapy, even though the beta-lactam is the superior drug. An even more extreme case would be if the patient were treated with daptomycin, an agent to which almost all isolates of $S$. aureus are susceptible, but that failed in a clinical trial of pneumonia treatment, likely due to inhibition by pulmonary surfactant [6]. Similarly, a patient with VAP caused by an extendedspectrum beta-lactamase-producing, gentamicin-susceptible pathogen, who received ceftazidime and gentamicin as empiric therapy at diagnosis of VAP, would also be considered to have received appropriate therapy according to the study definitions, although an aminoglycoside as monotherapy for pneumonia would not be considered clinically appropriate.

In the study, $37 \%$ of patients with VAP caused by MDR pathogens died within 30 days, versus $20 \%$ of patients with non-MDR VAP, a statistically significant difference $(p=0.02)$. The significance of MDR etiology is not sustained in multivariable analysis, leading the authors to conclude that MDR etiology is not an independent risk factor for mortality in VAP. Almost all patients in the MDR group received therapy considered appropriate according to the study definitions ( $79 \%$ by 24 hours and $87 \%$ by 48 hours). We therefore have only limited ability to determine the impact of resistance on mortality insofar as it causes delay in effective therapy. Although there were no differences between survivors and non-survivors in receipt of appropriate therapy at either 24 or 48 hours in this study, other studies, including a metaanalysis, have demonstrated such a difference [7]. Yet in the absence of data to distinguish between therapy that is clinically appropriate and therapy that is microbiologically sound but clinically problematic, the effect on mortality of delay in receiving truly appropriate therapy, and the potential impact of this delay on the results of the multivariable analysis, remain unknown.

As there is no ethically tenable way to compare outcomes of untreated MDR and non-MDR infection, studies that attempt to isolate the specific impact of resistance on outcome of infection cannot do so divorced from consideration of the effect of the therapy received. In addition to assessing delay in institution of therapy, analysis of the effect of appropriateness of therapy must take into account such issues as tissue penetration, pharmacokinetic and pharmacodynamic properties of the agent used, antimicrobial activity at varying $\mathrm{pH}$, and correctness of dosing and intervals of administration.

The outcomes study by Depuydt and colleagues, like others before it, addresses the question of the impact of resistance without dissecting fully the issue of appropriateness of therapy received. Future outcomes studies relating to infections with MDR pathogens must delve more deeply into this area in order to yield results that are less potentially confounded. Until such analyses are performed, we should consider the question of the impact of resistance on outcomes of bacterial infection as one for which more data are needed, but be guided by the abundance of evidence pointing toward a deleterious effect.

\section{Competing interests}

The authors declare that they have no competing interests.

\section{References}

1. Depuydt PO, Vandijck DM, Bekaert MA, Decruyenaere JM, Blot SI, Vogelaers DP, Benoit DD: Determinants and impact of multidrug antibiotic resistance in pathogens causing ventilatorassociated pneumonia. Crit Care 2008, 12:R142.

2. Combes A, Luyt CE, Fagon JY, Wolff M, Trouillet JL, Chastre J: Impact of piperacillin resistance on the outcome of Pseudomonas ventilator-associated pneumonia. Intensive Care Med 2006, 32:1970-1978.

3. Parker CM, Kutsogiannis J, Muscedere J, Cook D, Dodek P, Day AG, Heyland DK: Ventilator-associated pneumonia caused by multidrug-resistant organisms or Pseudomonas aeruginosa: prevalence, incidence, risk factors, and outcomes. J Crit Care 2008, 23:18-26.

4. Vidaur L, Planas K, Sierra R, Dimopoulos G, Ramirez A, Lisboa T, Rello J: Ventilator-associated pneumonia: impact of organisms on clinical resolution and medical resources utilization. Chest 2008, 133:625-632.

5. Shorr AF, Combes A, Kollef MH, Chastre J: Methicillin-resistant Staphylococcus aureus prolongs intensive care unit stay in ventilator-associated pneumonia, despite initially appropriate antibiotic therapy. Crit Care Med 2006, 34:700-706.

6. Maclayton DO, Hall RG 2nd: Pharmacologic treatment options for nosocomial pneumonia involving methicillin-resistant Staphylococcus aureus. Ann Pharmacother 2007, 41:235-244.

7. Kuti EL, Patel AA, Coleman Cl: Impact of inappropriate antibiotic therapy on mortality in patients with ventilator-associated pneumonia and blood stream infection: a meta-analysis. J Crit Care 2008, 23:91-100. 